\title{
ORIGINAL
}

\section{ENFERMEDADES PROFESIONALES DECLARADAS EN HOMBRES Y MUJERES EN ESPAÑA EN 2004}

\section{Montserrat García Gómez y Rosario Castañeda López}

Área de Salud Laboral. Subdirección General de Sanidad Ambiental y Salud Laboral. Dirección General de Salud Pública. Ministerio de Sanidad y Consumo.

\section{RESUMEN}

Fundamento: El conocimiento de la incidencia de las enfermedades derivadas del trabajo es un requisito esencial para la adopción de un enfoque racional de su control. La estadística oficial de las enfermedades profesionales no incluye la variable sexo. El objetivo de este estudio es describir las enfermedades profesionales reconocidas por el sistema de seguridad social español en el año 2004, buscando las diferencias en la morbilidad declarada entre hombres y mujeres.

Métodos: Se describe la información contenida en el Registro de Enfermedades Profesionales correspondiente al año 2004. Las variables incluidas son: sexo, edad, actividad económica, ocupación, antigüedad en el puesto de trabajo, tamaño de la empresa y Comunidad Autónoma. Como indicadores se han calculado porcentajes, tasas de incidencia brutas y específicas, y razón de tasas.

Resultados: En el año 2004 se notificaron 28.728 enfermedade profesionales. La incidencia en mujeres fue de 188,7 por 100.000 trabajadoras, y en hombres fue de 217,8 . La edad media de presentación en hombres fue de 39,4 \pm 11 años, y en mujeres de 37,6 \pm 11 años. El 52,7\% de las enfermedades se declaran en las mujeres en los 3 primeros años de permanencia en el puesto.

Conclusiones: Se constatan aspectos diferenciales de género en las enfermedades profesionales notificadas en España en el año 2004. Aunque la incidencia global es mayor en hombres que en mujeres, en la mayoría de actividades económicas y ocupaciones, las tasas específicas son mayores en mujeres. Son sobretodo las grandes empresas las que notifican enfermedades profesionales en las mujeres.

Palabras clave: Enfermedades profesionales. Diferencias de género. España.

\section{ABSTRACT}

Background: The knowledge of the occupational diseases incidence is an essential requisite for the adoption of rational control measures. The official statistics of the occupational diseases don't include the sex variable. The objective of this study is to describe occupational diseases recognized by the Spanish social security system in 2004 and assess the differences between men and women.

Methods: We describe the information of the Spanish Occupational Disease Registry notified in 2004. The considered variables are: sex, age, economic activity, occupation, and time in the workplace, size of the company and Autonomous Community. Percentages, crude and specific incidence rates per 100.000 workers and rates ratios have been computed as indicators.

Results: 28.728 occupational diseases were recorded in Spain in 2004. Women's incidence rate was 188,7 per 100.000 workers and 217,8 for men. The average age of occurrence was $39,4 \pm 11$ years for men and 37,6 \pm 11 years for women. The exposure time was lower than 3 years for $52,7 \%$ of the recorded diseases in women, and $44,6 \%$ in men.

Conclusions: We appreciate gender differences in the occupational diseases notified and recorded in Spain in 2004. The global incidence rate was higher in men, but the specific incidence rates in most of the economic activities and occupations were higher in women. Occupational diseases in women are mostly notified by big companies.

Key words: Occupational diseases. Gender differences. Spain.

Correspondencia:

Montserrat García Gómez

Área de Salud Laboral

Dirección General de Salud Pública

Ministerio de Sanidad y Consumo

Correo electrónico: mgarciag@msc.es 
En Alicia en el país de las maravillas, el sombrerero loco es un caso de enfermedad profesional. El término sombrerero loco data del siglo XIX, cuando los fabricantes de sombreros sufrieron los efectos del envenenamiento por mercurio, debido a la exposición a las sales de mercurio durante el proceso de producción. Cabe preguntarse ¿dónde están los sombrereros locos de hoy?

M. García Gómez.

\section{INTRODUCCIÓN}

El reconocimiento de que una afección va ligada a un riesgo laboral hace que se preste a tal riesgo una atención particular, lo que puede originar una mejora de la prevención, ya que se pone en evidencia el peligro, se preconizan medidas de protección y pueden ejercerse controles más eficaces cuando existe el riesgo ${ }^{1}$. En el caso de las enfermedades profesionales, el beneficio preventivo es además multiplicador ya que las exposiciones origen del daño afectan no sólo a la persona enferma sino también a las otras que desempeñan tareas similares en el mismo entorno.

Existen razones para pensar que en España, como en otros países, existe una infraestimación del número de enfermedades profesionales. En comparación con otros países de nuestro entorno y salvando las diferencias en los sistemas de notificación de cada país, las cifras españolas respecto a este tema son sensiblemente inferiores (entre dos y diez veces) a las estimaciones de la mayoría de los países de la $\mathrm{OCDE}^{2}$. Algunos autores estiman que los sistemas de indemnización por enfermedad profesional y accidente de trabajo compensan menos del $10 \%$ de los casos de enfermedad profesional ${ }^{3} \mathrm{y}$, en la mayoría de los casos, se trata de enfermedades leves, como la dermatitis.

Si conociésemos la realidad de las cifras de enfermedades derivadas del trabajo nos sorprendería comprobar que son mayores que las de los accidentes ${ }^{4,5}$. Algunos autores aducen que, dado que conocemos muy bien los principios de la prevención primaria, lo importante es concentrar los esfuerzos en ella, eliminando o controlando las exposiciones nocivas en los centros de trabajo, dejando el sistema de indemnización por enfermedad profesional circunscrito a la recaudación y asignación de fondos destinados al pago de las prestaciones ${ }^{3}$.

Sin embargo, el conocimiento de la incidencia y prevalencia de las enfermedades derivadas del trabajo mediante un procedimiento fiable y exhaustivo, es un requisito esencial para la adopción de un enfoque racional del control de las enfermedades y lesiones profesionales. Sólo mediante una evaluación epidemiológica de las dimensiones de una enfermedad profesional se puede determinar su importancia relativa dentro de los problemas de salud pública, la necesidad de recursos, las tendencias en diferentes grupos, lugares y periodos, en resumen, el establecimiento de prioridades y estrategias de control, investigación y evaluación de cualquier intervención que se emprenda. No se pueden diseñar políticas y planes de salud laboral eficaces sin conocer la totalidad del problema.

Por otro lado, la descripción y análisis oficiales de las enfermedades profesionales no incluyen la variable sexo, lo que incrementa la carencia de conocimiento de las mismas, ya que impide incorporar la perspectiva de género al análisis del problema. Efectivamente, las encuestas europeas y españolas de condiciones de trabajo ${ }^{6,7}$ muestran que mientras los hombres tienen trabajos que suelen requerir mayor esfuerzo físico, y están expuestos a un mayor número de factores clásicos (químicos, físicos, accidentes, 
etc.), entre los factores de riesgo más prevalentes de las mujeres trabajadoras encontramos el alto nivel de exigencia, la monotonía, el sedentarismo, las posturas forzadas, la necesidad de rapidez y destreza en el puesto de trabajo, la poca cualificación y responsabilidad, la acumulación de tareas, y la inseguridad de mantenimiento del puesto. Sabemos que existen sectores de empleo claramente feminizados y también que, en los no feminizados, se produce una división de tareas según el sexo de las y los trabajadores. Según la V Encuesta Nacional de Condiciones de Trabajo $^{7}$ (V ENCT) tanto las mujeres trabajadoras en el sector industrial como las trabajadoras del sector servicios presentan, en comparación con los hombres, mayor frecuencia de cansancio y cefalea; entre las mujeres de servicios, y en relación a los hombres, existe una mayor prevalencia de irritabilidad y molestias visuales; además, las mujeres han acudido con mayor frecuencia que los hombres a consulta médica por un problema de salud relacionado con el trabajo: el motivo de consulta más frecuente fue el dolor de espalda ${ }^{8}$.

A pesar de todas estas evidencias, con mucha frecuencia se excluye a las mujeres en los estudios de salud laboral y, como hemos dicho, no se incluye el sexo como variable en las estadísticas de siniestralidad laboral, ni en el análisis de los accidentes de trabajo ni en el de las enfermedades profesionales.

El objetivo de este trabajo es describir las enfermedades profesionales reconocidas por el sistema de seguridad social español en el año 2004 buscando las diferencias en los perfiles de morbilidad laboral declarada entre hombres y mujeres.

\section{MATERIAL Y MÉTODOS}

Se describe la información contenida en los partes de declaración de enfermedad profesional, facilitada por la Subdirección
General de Estadísticas Sociales y Laborales del Ministerio de Trabajo y Asuntos Sociales, en formato electrónico, del año 2004. Las variables de interés contenidas en el parte y utilizadas en este análisis han sido:

- del trabajador: sexo, edad, ocupación (Clasificación Nacional de Ocupaciones ${ }^{9}$ ), tiempo en el puesto de trabajo y Comunidad Autónoma.

- de la empresa: actividad económica (Clasificación Nacional de Actividades Económicas ${ }^{10}$ ) y tamaño de la empresa.

- de la enfermedad: diagnostico, según la Clasificación Internacional de Enfermedades ${ }^{11}$.

Se han utilizado como indicadores los porcentajes, las tasas de incidencia brutas y específicas por cien mil trabajadores asalariados (equivalentes a índices de incidencia en la normativa laboral) para cada una de las variables incluidas en el estudio, y la razón de tasas con los intervalos de confianza al 95\%. Para calcular las tasas de incidencia se ha utilizado como denominador la población asalariada por género, edad, rama de actividad económica, ocupación y Comunidad Autónoma, de la Encuesta de Población Activa (EPA) ${ }^{12}$.

\section{RESULTADOS}

En el año 2004 se notificaron en España un total de 28.728 enfermedades profesionales (EP), correspondiendo el $62,4 \%$ a hombres y el $37,6 \%$ a mujeres. La incidencia en las mujeres fue de 188,7 enfermedades profesionales por 100.000 trabajadoras, y en los hombres fue de 217,8. La razón de incidencias hombre/mujer fue de 1,15 (tabla 1).

Si tenemos en cuenta la edad, la edad media de presentación en los hombres fue de 
Tabla 1

Enfermedades profesionales notificadas en España en 2004 por sexo

\begin{tabular}{|l|c|c|c|c|c|c|}
\hline \multicolumn{1}{|c|}{ Enfermedad } & $\begin{array}{c}\mathbf{N}^{\mathbf{0}} \mathbf{E P} \\
\text { Mujer }\end{array}$ & II Mujer & $\begin{array}{c}\mathbf{N}^{\mathbf{}} \mathbf{E P} \\
\text { Hombre }\end{array}$ & II Hombre & $\begin{array}{c}\text { Razón de tasas } \\
\text { M/H (IC 95\%) }\end{array}$ & $\begin{array}{c}\text { Razón de tasas } \\
\text { H/M (IC 95\%) }\end{array}$ \\
\hline Infecciosas & 156 & 2,72 & 173 & 2,1 & $1,30(1,04-1,61)$ & \\
\hline Cáncer & 1 & 0,02 & 8 & 0,1 & & $5(0,89-28,14)$ \\
\hline Neurológicas & 1.787 & 31,19 & 1.525 & 18,54 & $1,68(1,57-1,80)$ & \\
\hline De los órganos de los sentidos & 64 & 1,12 & 580 & 7,05 & & $6,29(5,03-7,88)$ \\
\hline Cardiovasculares & 2 & 0,03 & 11 & 0,13 & & $4,33(0,94-19,97)$ \\
\hline Respiratorias & 148 & 2,58 & 340 & 4,13 & & $1,60(1,32-1,94)$ \\
\hline Cutáneas & 1.309 & 22,85 & 2.304 & 28,01 & & $1,23(1,15-1,31)$ \\
\hline Osteomusculares & 7.344 & 128,2 & 12,976 & 157,73 & & $1,23(1,20-1,27)$ \\
\hline Total & 10.811 & 188,72 & 17.917 & 217,79 & & $1,15(1,13-1,18)$ \\
\hline
\end{tabular}

EP: enfermedades profesionales. II: Índice de incidencia expresado en $\mathrm{n}^{\circ}$ de EP por 100.000 asalariados. M/H: Mujer/Hombre. IC 95\%: Intervalo de confianza al 95\%.

\section{Figura 1}

Enfermedades profesionales notificadas en España en 2004 por grupos de edad y sexo
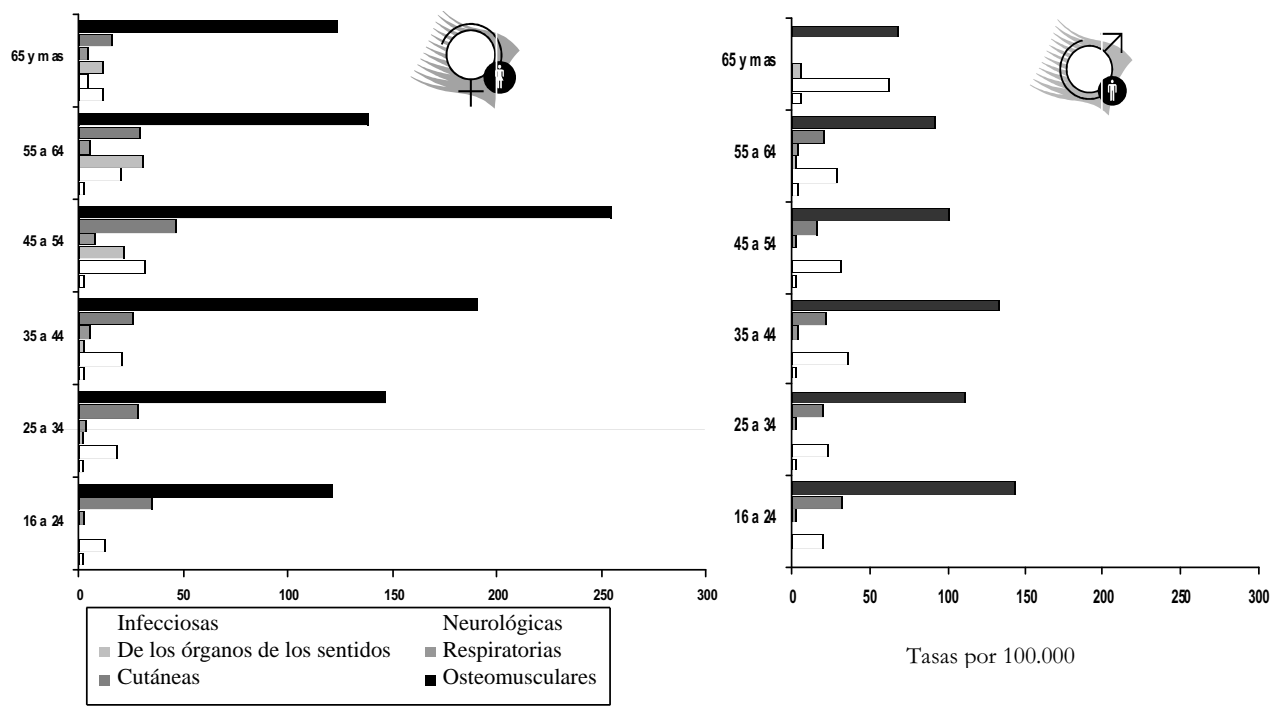

39,4 11 años, y en las mujeres de 37,6 \pm 11

143,16 respectivamente, y las neurológicas años. Las enfermedades más prevalentes, cutáneas y osteomusculares, se declaran en las mujeres con mayor frecuencia en el grupo de 16 a 24 años con tasas de 32,19 y en el grupo de 35 a 44 años, con una tasa de 36,15 , mientras que en los hombres la incidencia mayor se observa en el grupo de 45 a 54 años (figura 1). 
Tabla 2

Actividades económicas en las que se acumularon más enfermedades profesionales notificadas en España en 2004 por sexo

\begin{tabular}{|c|c|c|c|c|}
\hline Actividad & II Mujer & II Hombre & $\begin{array}{l}\text { Razón de Tasas } \\
\text { M/H (IC } 95 \%)\end{array}$ & $\begin{array}{l}\text { Razón de Tasas } \\
\text { H/M (IC } 95 \%)\end{array}$ \\
\hline Agricultura, ganadería, caza y selvicultura & 229,09 & 107,59 & $2,13(1,81-2,50)$ & \\
\hline Agricultura & 230,55 & 91,45 & $2,52(2,13-2,98)$ & \\
\hline Pesca & 90,91 & 271,43 & & $2,99(0,46-19,46)$ \\
\hline Industrias Extractivas & 2745,5 & 2609,5 & $1,05(0,89-1,24)$ & \\
\hline Extracción de productos energéticos & 314,29 & 4574,26 & & $14,55(9,40-22,53)$ \\
\hline Extracción de otros minerales & 7368,42 & 1206,41 & $6,11(5,18-7,20)$ & \\
\hline Industrias Manufactureras & 705,98 & 477,16 & $1,45(1,43-1,53)$ & \\
\hline Industria de la alimentación bebidas y tabaco & 925,45 & 647,55 & $1,43(1,33-1,54)$ & \\
\hline Industria textil y de la confección & 396,85 & 201,98 & $1,96(1,63-2,36)$ & \\
\hline Industria del cuero y del calzado & 395,06 & 519,77 & & $1,32(1,05-1,65)$ \\
\hline Industria de la madera y del corcho & 485,29 & 430,40 & $1,13(0,87-1,47)$ & \\
\hline Industria del papel. Edición y artes graficas & 237,74 & 217,48 & $1,09(0,90-1,32)$ & \\
\hline Coquería, y refinado de petróleo & 277,78 & 26,32 & $10,56(3,69-30,22)$ & \\
\hline Industria química & 455,02 & 301,93 & $1,51(1,28-1,78)$ & \\
\hline Industria de transformación del caucho y materiales plástico & 1923,77 & 564,10 & $3,41(3,02-3,85)$ & \\
\hline Industrias de otros productos minerales no metálicos & 483,47 & 306,19 & $1,58(1,29-1,93)$ & \\
\hline Metalurgia y fabricación de productos metálicos & 962,88 & 698,92 & $1,38(1,25-1,52)$ & \\
\hline Industria de construcción de maquinaria y equipos metálicos & 743,70 & 480,10 & $1,55(1,32-1,82)$ & \\
\hline Industria de material y equipo eléctrico, electrónico y óptico & 803,42 & 241,95 & $3,32(2,86-3,85)$ & \\
\hline Fabricación de automóviles y remolques & 1400,41 & 577,56 & $2,42(2,22-2,64)$ & \\
\hline Fabricación de muebles. Reciclaje & 502,84 & 343,92 & $1,46(1,23-1,73)$ & \\
\hline Producción y distribución de energía eléctrica, gas y agua & 15,71 & 66,91 & & $4,26(1,47-12,37)$ \\
\hline Construcción & 84,38 & 233,21 & & $2,76(2,24-3,41)$ \\
\hline Comercio & 147,41 & 139,76 & $1,05(1,00-1,16)$ & \\
\hline Venta y reparación de vehículos de motor & 104,74 & 188,35 & & $1,80(1,31-2,45)$ \\
\hline Comercio al por mayor e intermediación & 190,98 & 137,91 & $1,38(1,20-1,59)$ & \\
\hline Comercio al por menor & 139,91 & 104,05 & $1,34(1,20-1,50)$ & \\
\hline Hostelería & 190,74 & 69,90 & $2,73(2,28-3,13)$ & \\
\hline Transporte, almacenamiento y comunicaciones & 100,05 & 84,38 & $1,19(1,01-1,39)$ & \\
\hline Transporte terrestre, marítimo y aéreo & 67,63 & 81,95 & & $1,21(0,80-1,67)$ \\
\hline Actividades de Agencias de viajes, & 272,54 & 208,15 & $1,31(1,05-1,63)$ & \\
\hline Correos y telecomunicaciones & 36,66 & 13,14 & $2,79(1,64-4,75)$ & \\
\hline Intermediación financiera y seguros & 30,93 & 5,52 & $5,61(3,19-9,85)$ & \\
\hline Actividades inmobiliarias y de alquiler & 209,71 & 89,62 & $2,34(2,12-2,59)$ & \\
\hline Actividades inmobiliarias & 22,54 & 46,88 & & $2,08(0,97-4,45)$ \\
\hline $\begin{array}{l}\text { Alquiler de máquinas y equipos, de efectos personales y enseres } \\
\text { domésticos }\end{array}$ & 18,35 & 179,37 & & $9,78(3,09-30,95)$ \\
\hline Actividades informáticas e investigación & 48,39 & 18,20 & $2,66(1,50-4,70)$ & \\
\hline Actividades jurídicas, de contabilidad, auditorias, asesoría fiscal & 246,64 & 108,73 & $2,27(2,04-2,53)$ & \\
\hline Administración Pública, Defensa y Seguridad Social & 58,19 & 42,43 & $1,37(1,16-1,62)$ & \\
\hline Educación & 16,83 & 9,80 & $1,72(1,16-2,54)$ & \\
\hline Actividades sanitarias y veterinarias. Servicios Sociales & 131,96 & 72,84 & $1,81(1,13-2,13)$ & \\
\hline Otras actividades sociales y servicios prestados a la comunidad & 190,46 & 106 & $1,80(1,56-2,13)$ & \\
\hline Actividades de saneamiento público & 652,78 & 205,17 & $3,18(2,31-4,37)$ & \\
\hline Actividades asociativas & 49,75 & 24,86 & $2,00(0,93-4,33)$ & \\
\hline Actividades recreativas, culturales y deportivas & 83,75 & 68,71 & $1,22(0,93-1,60)$ & \\
\hline Actividades diversas de servicios personales & 329,94 & 237,50 & $1,39(1,05-1,83)$ & \\
\hline Actividades de los hogares & 1,72 & 28,93 & & $16,78(1,05-1,83)$ \\
\hline Total & 188,72 & 217,79 & & $1,15(1,13-1,18)$ \\
\hline
\end{tabular}

EP: enfermedades profesionales. II: Índice de incidencia expresado en $\mathrm{n}^{\circ}$ de EP por 100.000 asalariados. M/H: Mujer/Hombre. IC 95\%: Intervalo de confianza al 95\% 
Tabla 3

Ocupaciones en las que se acumularon más enfermedades profesionales notificadas en España en 2004 por sexo

\begin{tabular}{|c|c|c|c|}
\hline Mujeres & $\mathbf{N}^{0} \mathbf{E P}$ & II Mujer & $\begin{array}{l}\text { Razón de Tasas } \\
\text { M/H (IC 95\%) }\end{array}$ \\
\hline Total ocupaciones & 10811 & 188,72 & \\
\hline Trabajadoras no cualificadas & 3800 & 328,46 & \\
\hline $\begin{array}{l}\text { Artesanas y trabajadoras cualificadas de industrias manufactureras, construcción y minería, } \\
\text { excepto operadoras }\end{array}$ & 2561 & 1570,2 & $3,4(3,26-3,53)$ \\
\hline Trabajadoras de servicios de restauración, personales, protección y vendedoras de comercio & 2328 & 171,67 & $2,1(1,97-2,33)$ \\
\hline Operadoras de instalaciones y maquinaria, montadoras & 916 & 456,18 & $2,3(2,18-2,53)$ \\
\hline Empleadas de tipo administrativo & 470 & 47,56 & $1,4(1,18-1,66)$ \\
\hline Técnicas y profesionales científicas e intelectuales & 327 & 32,18 & $2,2(1,88-2,77)$ \\
\hline Técnicas y profesionales de apoyo & 316 & 41,9 & $1,5(1,30-1,81)$ \\
\hline Trabajadoras cualificadas en agricultura y pesca & 87 & 462,77 & $2,5(1,98-3,16)$ \\
\hline Dirección de las empresas y de la Administración Pública & 6 & 9,6 & $1,6(0,63-4,26)$ \\
\hline Hombres & $\mathbf{N}^{\circ} \mathbf{E P}$ & II Hombre & $\begin{array}{l}\text { Razón de Tasas } \\
\text { M/H (IC 95\%) }\end{array}$ \\
\hline Total ocupaciones & 17917 & 217,78 & $1,15(1,13-1,18)$ \\
\hline $\begin{array}{l}\text { Artesanos y trabajadores cualificados de industrias manufactureras, construcción y minería, } \\
\text { excepto operadoras }\end{array}$ & 10036 & 462,7 & \\
\hline Trabajadores no cualificados & 3910 & 343,49 & $1,05(1,00-1,09)$ \\
\hline Operadores de instalaciones y maquinaria, montadores & 2443 & 194,24 & \\
\hline Trabajadores de servicios de restauración, personales, protección y vendedores de comercio & 686 & 80,06 & \\
\hline Trabajadores cualificados en agricultura y pesca & 270 & 184,8 & \\
\hline Técnicos y profesionales de apoyo & 245 & 27,28 & \\
\hline Empleados de tipo administrativo & 183 & 34,04 & \\
\hline Técnicos y profesionales científicos e intelectuales & 131 & 14,14 & \\
\hline Dirección de las empresas y de la Administración Pública & 13 & 5,86 & \\
\hline
\end{tabular}

EP: enfermedades profesionales. II: Índice de incidencia expresado en $\mathrm{n}^{\mathrm{o}}$ de EP por 100.000 asalariados. M/H: Mujer/Hombre. IC 95\%: Intervalo de confianza al 95\%.

Por lo que se refiere al sector de actividad económica, tal y como podemos ver en la tabla 2, destacan las Industrias Extractivas, con una tasa de 2.609,5 en los hombres y 2.745,5 en las mujeres. En las Manufactureras la tasa en mujeres es de 705,98 frente a 477,16 en los hombres. Dentro de ellas, la Industria de transformación del caucho y la Fabricación de automóviles, son las que presentan mayor incidencia en las mujeres (tasas de $1.923,77$ en la primera y 1.400,41).
En la tabla 3 se presentan las incidencias de las enfermedades profesionales por ocupación.

Otra variable a tener en cuenta es el tiempo de exposición al riesgo. En el 47,65\% de las enfermedades notificadas, el tiempo de exposición fue menor de 3 años, y en un $19,51 \%$ menor a 6 meses (figura 2). La media de antigüedad en el conjunto de los procesos fue de 8,5 $\pm 10,3$ años en los hombres, y de 6,5 $\pm 9,1$ años en las mujeres. 
Figura 2

Antigüedad en el puesto de trtabajo de los trabajadores con enfermedad profesional notificada en España en 2004

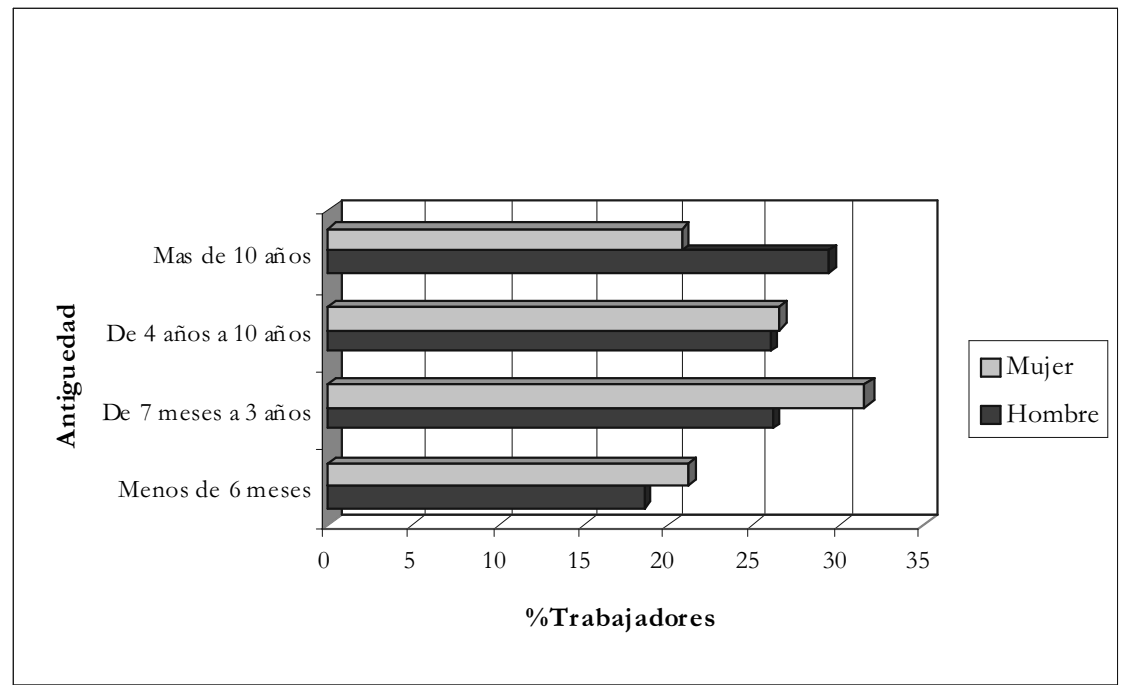

Figura 3

Enfermedades profesionales notificadas en España en 2004 por Comunidad Autónoma y sexo

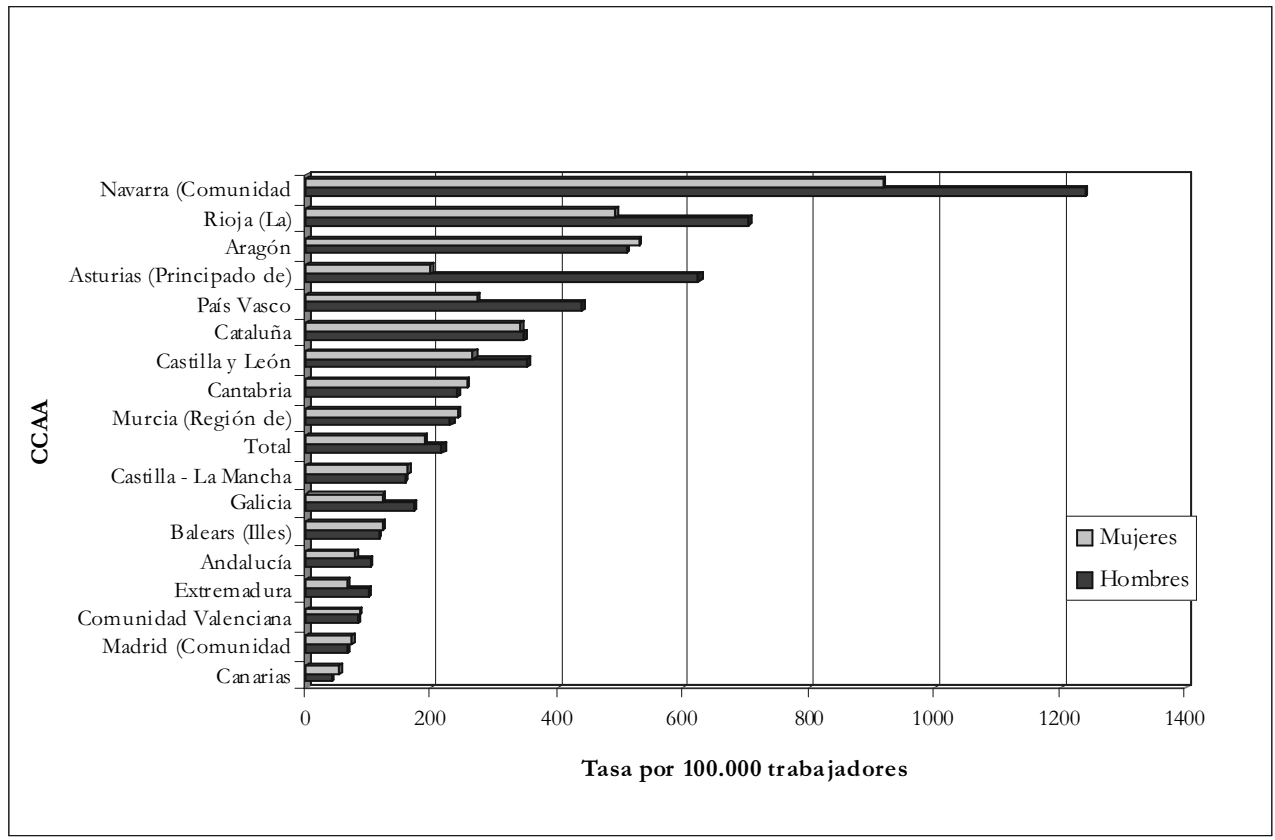




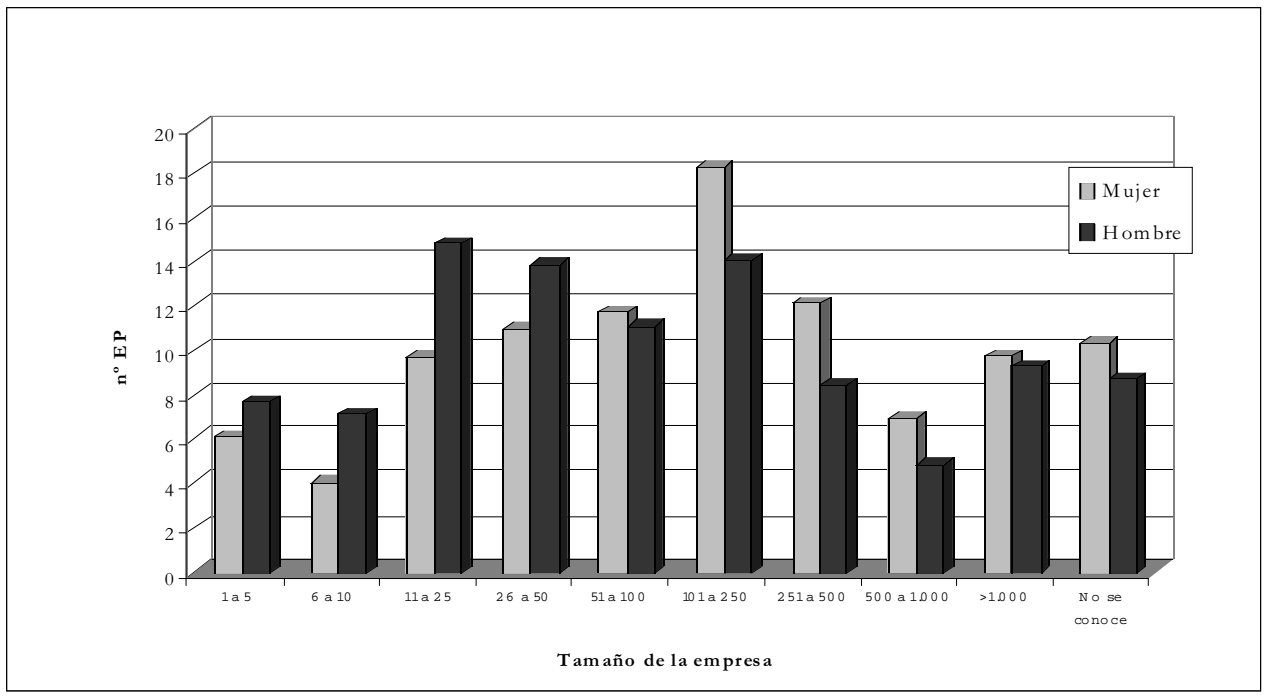

Respecto a su distribución geográfica, observamos que las Comunidades Autónomas que presentan mayor incidencia son: Navarra, con 1.100,5 enfermedades profesionales por cien mil trabajadores $(917,5$ en mujeres y 1.237,9 en hombres), la Rioja, Aragón y Asturias, con una incidencia mayor del doble que la media (figura 3 ).

En cuanto al tamaño de las empresas, se ha observado el $52 \%$ de casos de enfermedad en las que tienen más de 50 trabajadores, sobre todo en lo que se refiere a las mujeres (figura 4).

Para completar el análisis que venimos realizando y profundizar en la mirada de género, describimos a continuación las principales características de las enfermedades más prevalentes, tanto en hombres como en mujeres en las tablas 4 y 5 . En estas tablas se describen por grupo de enfermedad, de mayor a menor frecuencia de declaración, estas enfermedades.
La tenosinovitis de mano y muñeca presenta en mujeres una tasa de 74,42. Es un $44 \%$ mas frecuente que las epicondilitis y 2,39 veces mayor que el túnel carpiano. En las mujeres la incidencia es cinco veces mayor que en los hombres en la Industria de material y equipo eléctrico, electrónico y óptico. Por ocupación, las mujeres artesanas y trabajadoras cualificadas de Industrias manufactureras, construcción y minería con una tasa de 668,91 y los trabajadores no cualificados con 135,03, son las que presentan una mayor incidencia.

Las epicondilitis son las enfermedades mas frecuentes en los hombres. Son un $11 \%$ mas frecuentes que la tenosinovitis de mano y muñeca, y 3,85 veces mayor que la dermatitis alérgica de contacto. Las mujeres que trabajan en la industria de transformación del caucho con una tasa de 547,09, en Material y equipos eléctricos con 254,27 y en la Hostelería con 55,05, presentan una incidencia que es mayor del doble que en hombres. 
Tabla 4

Comparación de incidencias de las principales enfermedades profesionales notificadas en España en 2004 por actividad y sexo

\begin{tabular}{|c|c|c|c|}
\hline Enfermedad & Actividad económica & $\begin{array}{l}\text { Razón de tasas } \\
\text { M/H (IC 95\%) }\end{array}$ & $\begin{array}{l}\text { Razón de tasas } \\
\text { H/M (IC 95\%) }\end{array}$ \\
\hline \multirow{13}{*}{$\begin{array}{l}\text { Tenosinovitis de mano- } \\
\text { muñeca }\end{array}$} & Total & $1,10(1,06-1,15)$ & \\
\hline & Industria de material y equipo eléctrico, electrónico y óptico & $5,06(3,96-6,44)$ & \\
\hline & Transformación del caucho y materias plásticas & $3,23(2,67-3,91)$ & \\
\hline & \begin{tabular}{|l} 
Fabricación de material de transporte \\
\end{tabular} & $3,15(2,76-3,60)$ & \\
\hline & Construcción de maquinaria y equipo mecánico & $2,88(2,30-3,61)$ & \\
\hline & Hostelería & $2,67(2,12-3,37)$ & \\
\hline & Construcción & & $2,65(1,79-3,90)$ \\
\hline & Industria textil y de la confección & $2,31(1,96-3,23)$ & \\
\hline & Actividades sanitarias y veterinarias, servicios sociales & $2,24(1,62-3,09)$ & \\
\hline & Metalurgia y fabricación de productos metálicos & $1,94(1,66-2,27)$ & \\
\hline & Comercio al por mayor e intermediarios del comercio & $1,64(1,32-2,03)$ & \\
\hline & Comercio al por menor y reparaciones domésticas & $1,54(1,29-1,85)$ & \\
\hline & Industria de la alimentación, bebidas y tabaco & $1,53(1,38-1,70)$ & \\
\hline \multirow[t]{9}{*}{ Epicondilitis } & Total & & $1,46(1,40-1,52)$ \\
\hline & Extracción de productos energéticos & & $10,36(2,46-5,41)$ \\
\hline & Construcción & & $3,65(1,79-3,90)$ \\
\hline & Transformación del caucho y materias plásticas & $3,31(2,63-4,15)$ & \\
\hline & Hostelería & $2,31(1,81-2,94)$ & \\
\hline & Fabricación de material de transporte & $2,17(1,85-2,55)$ & \\
\hline & Venta y reparación de vehículos, venta al por menor de combustible & & $2,13(1,25-3,62)$ \\
\hline & Industria textil y de la confección & $1,71(1,26-2,32)$ & \\
\hline & Actividades sanitarias y veterinarias, servicios sociales & $1,71(1,26-2,32)$ & \\
\hline \multirow{10}{*}{$\begin{array}{l}\text { Síndrome del túnel } \\
\text { carpiano }\end{array}$} & Total & $1,68(1,57-1,80)$ & \\
\hline & Fabricación de material de transporte & $5,93(4,69-7,48)$ & \\
\hline & Hostelería & $5,21(4,69-7,48)$ & \\
\hline & \begin{tabular}{|l|} 
Transformación del caucho y materias plásticas \\
\end{tabular} & $4,92(4,92-7,05)$ & \\
\hline & Industria textil y de la confección & $3,29(1,99-5,44)$ & \\
\hline & Actividades sanitarias y veterinarias, servicios sociales & $2,83(2,38-9,14)$ & \\
\hline & Comercio al por menor y reparaciones domésticas & $2,79(2,01-3,87)$ & \\
\hline & Metalurgia y fabricación de productos metálicos & $2,30(1,75-3,03)$ & \\
\hline & Industria de la alimentación, bebidas y tabaco & $2,29(1,90-2,77)$ & \\
\hline & Construcción & & $1,66(0,92-3,01)$ \\
\hline \multirow[t]{4}{*}{ Bursitis de la rodilla } & Total & & $4,67(3,81-5,73)$ \\
\hline & Metalurgia y fabricación de productos metálicos & & $3,42(0,91-12,91)$ \\
\hline & Construcción & & $2,35(0,91-12,91)$ \\
\hline & Comercio al por menor y reparaciones domésticas & & $2,03(0,91-4,52)$ \\
\hline \multirow[t]{9}{*}{ Dermatitis } & Total & & $1,22(1,14-1,31)$ \\
\hline & Venta y reparación de vehículos, venta al por menor de combustible & & $7,01(2,10-23,32)$ \\
\hline & Transformación del caucho y materias plásticas & $3,79(2,73-5,26)$ & \\
\hline & Construcción & & $2,6(2,10-23,32)$ \\
\hline & \begin{tabular}{|l|} 
Hostelería \\
\end{tabular} & $2,58(1,84-3,62)$ & \\
\hline & Actividades sanitarias y veterinarias, servicios sociales & $2,16(1,41-3,31)$ & \\
\hline & \begin{tabular}{|l} 
Fabricación de material de transporte \\
\end{tabular} & $1,85(1,38-2,45)$ & \\
\hline & Industria química & & $1,61(1,11-2,33)$ \\
\hline & Industria textil y de la confección & $1,26(1,38-2,45)$ & \\
\hline \multirow[t]{4}{*}{ Asma } & Total & & $1,49(1,19-1,87)$ \\
\hline & Agricultura, ganadería, caza y silvicultura & $4,1(1,28-13,15)$ & \\
\hline & Industria química & & $3,71(0,92-14,98)$ \\
\hline & Industria de la alimentación, bebidas y tabaco & & $3,15(1,55-6,40)$ \\
\hline \multirow{2}{*}{$\begin{array}{l}\text { Hepatitis de transmisión } \\
\text { sanguínea }\end{array}$} & Total & $1,57(1,40-1,77)$ & \\
\hline & Actividades sanitarias y veterinarias, servicios sociales & $1,38(0,85-2,24)$ & \\
\hline Tuberculosis & Total & $2,21(1,34-3,65)$ & \\
\hline \multirow[t]{4}{*}{ Sordera } & Total & & $6,39(5,10-8,01)$ \\
\hline & Fabricación de material de transporte & & $10,3(4,11-25,81)$ \\
\hline & Metalurgia y fabricación de productos metálicos & & $3,44(4,11-25,81)$ \\
\hline & Industria de la alimentación, bebidas y tabaco & $1,9(0,92-3,94)$ & \\
\hline
\end{tabular}

EP: enfermedades profesionales. II: Índice de incidencia expresado en $\mathrm{n}^{\circ}$ de EP por 100.000 asalariados. M/H: Mujer/Hombre. IC 95\%: Intervalo de confianza al 95\% 
Tabla 5

Comparación de incidencias de las principales enfermedades profesionales notificadas en España en 2004 por ocupación y sexo

\begin{tabular}{|c|c|c|c|}
\hline Enfermedad & Ocupación & $\begin{array}{l}\text { Razón de tasas } \\
\text { M/H (IC 95\%) }\end{array}$ & $\begin{array}{l}\text { Razón de tasas } \\
\text { H/M (IC 95\%) }\end{array}$ \\
\hline \multirow{8}{*}{$\begin{array}{l}\text { Tenosinovitis de mano- } \\
\text { muñeca }\end{array}$} & Total & $1,10(1,06-1,15)$ & \\
\hline & Artesanos y trabajadores cualificados de industrias manufactureras, construcción y minería & $5,23(4,91-5,57)$ & \\
\hline & Técnicos y profesionales científicos e intelectuales (Físicos, químicos, médicos, enfermeros.....) & $2,92(1,96-4,35)$ & \\
\hline & Operadores de instalaciones y maquinaria, montadores & $2,66(2,35-3,01)$ & \\
\hline & Trabajadores cualificados en agricultura y pesca & $2,64(1,81-3,86)$ & \\
\hline & Trabajadores de servicios de restauración, personales, protección y vendedores de comercio & $2,50(2,16-2,89)$ & \\
\hline & Empleados administrativos & $2,47(1,82-3,35)$ & \\
\hline & Técnicos y profesionales de apoyo & $2,03(1,52-2,72)$ & \\
\hline \multirow[t]{6}{*}{ Epicondilitis } & Total & & $1,46(1,40-1,52)$ \\
\hline & Artesanos y trabajadores cualificados de industrias manufactureras, construcción y minería & $2,60(1,91-3,53)$ & \\
\hline & Trabajadores cualificados en agricultura y pesca & $2,23(1,12-4,45)$ & \\
\hline & Técnicos y profesionales científicos e intelectuales (Físicos, químicos, médicos, enfermeros.....) & $2,23(2,02-2,45)$ & \\
\hline & Operadores de instalaciones y maquinaria, montadores & $2,14(1,76-2,59)$ & \\
\hline & \begin{tabular}{|l} 
Trabajadores de servicios de restauración, personales, protección y vendedores de comercio \\
\end{tabular} & $1,63(1,60-1,67)$ & \\
\hline \multirow[t]{7}{*}{ Síndrome del túnel carpiano } & Total & $1,68(1,57-1,80)$ & \\
\hline & Artesanos y trabajadores cualificados de industrias manufactureras, construcción y minería & $7,22(6,54-7,96)$ & \\
\hline & Trabajadores cualificados en agricultura y pesca & $5,05(2,70-9,45)$ & \\
\hline & Operadores de instalaciones y maquinaria, montadores & $4,30(3,53-5,23)$ & \\
\hline & Trabajadores de servicios de restauración, personales, protección y vendedores de comercio & $3,41(2,67-4,35)$ & \\
\hline & Empleados administrativos & $3,08(1,83-5,19)$ & \\
\hline & Técnicos y profesionales científicos e intelectuales (Físicos, químicos, médicos, enfermeros.....) & $2,96(1,39-6,30)$ & \\
\hline \multirow[t]{3}{*}{ Bursitis de la rodilla } & Total & & $4,67(3,81-5,73)$ \\
\hline & Trabajadores no cualificados & & $2,88(1,94-4,28)$ \\
\hline & Artesanos y trabajadores cualificados de industrias manufactureras, construcción y minería & & $1,95(1,21-3,13)$ \\
\hline \multirow[t]{7}{*}{ Dermatitis } & Total & & $1,22(1,14-1,31)$ \\
\hline & Artesanos y trabajadores cualificados de industrias manufactureras, construcción y minería & $2,69(2,35-3,08)$ & \\
\hline & Trabajadores cualificados en agricultura y pesca & $2,59(1,14-5,90)$ & \\
\hline & Trabajadores de servicios de restauración, personales, protección y vendedores de comercio & $2,51(2,01-3,13)$ & \\
\hline & Trabajadores no cualificados & & $1,35(1,20-1,53)$ \\
\hline & Técnicos y profesionales científicos e intelectuales (Físicos, químicos, médicos, enfermeros.....) & $2,11(1,30-3,44)$ & \\
\hline & Operadores de instalaciones y maquinaria, montadores & $2,04(1,64-2,54)$ & \\
\hline \multirow[t]{4}{*}{ Asma } & Total & & $1,49(1,19-1,87)$ \\
\hline & Artesanos y trabajadores cualificados de industrias manufactureras, construcción y minera & $2,46(1,58-3,82)$ & \\
\hline & Operadores de instalaciones y maquinaria, montadores & $1,61(0,81-3,20)$ & \\
\hline & Trabajadores de servicios de restauración, personales, protección y vendedores de comercio & & $1,59(0,87-2,88)$ \\
\hline \multirow{3}{*}{$\begin{array}{l}\text { Hepatitis de transmisión } \\
\text { sanguínea }\end{array}$} & Total & $4,31(2,94-6,29)$ & \\
\hline & Técnicos y profesionales científicos e intelectuales (Físicos, químicos, médicos, enfermeros.....) & $5,93(3,08-11,40)$ & \\
\hline & Trabajadores de servicios de restauración, personales, protección y vendedores de comercio & $1,59(0,81-3,07)$ & \\
\hline \multirow[t]{3}{*}{ Tuberculosis } & Total & $2,21(1,34-3,65)$ & \\
\hline & Técnicos y profesionales científicos e intelectuales (Físicos, químicos, médicos, enfermeros.....) & $1,37(0,62-3,03)$ & \\
\hline & Trabajadores de servicios de restauración, personales, protección y vendedores de comercio & $3,16(1,14-8,73)$ & \\
\hline \multirow[t]{4}{*}{ Sordera } & Total & & $6,39(5,10-8,01)$ \\
\hline & Operadores de instalaciones y maquinaria, montadores & & $2,58(1,17-5,71)$ \\
\hline & Trabajadores no cualificados & & $2,3(1,37-2,30)$ \\
\hline & Artesanos y trabajadores cualificados de industrias manufactureras, construcción y minera & & $1,89(1,16-3,09)$ \\
\hline
\end{tabular}

EP: enfermedades profesionales. II: Índice de incidencia expresado en $\mathrm{n}^{\circ}$ de EP por 100.000 asalariados. M/H: Mujer/Hombre. IC 95\%: Intervalo de confianza al 95\%

Por ocupación, las epicondilitis presentan una mayor incidencia en las artesanas y trabajadoras cualificadas de manufacturas, en las ope- radoras de instalaciones y maquinaria y en las cualificadas de la agricultura con tasas de $431,64,153,39$ y 143,62 respectivamente. 
La dermatitis presenta mayor incidencia en hombres que en mujeres (razón 1,22, IC 95\% 1,14-1,31). En las mujeres, la tasa duplica a la de los hombres en las Actividades sanitarias y veterinarias, Hostelería, y Servicios personales. Por ocupación, las artesanas y trabajadoras cualificadas de la industria manufacturera, y las trabajadoras de servicios de restauración y personales, son las que presentan una mayor incidencia.

El túnel carpiano presenta una mayor incidencia en mujeres que en hombres (razón 1,68 , IC 95\% 1,57-1,80), globalmente y en todas las ramas de actividad, con la excepción de aquellas actividades donde la presencia femenina es escasa (Construcción, Producción y distribución de energía eléctrica y gas, y Extracción de productos energéticos).

La frecuencia de notificación del asma es baja, se han notificado 227 casos en hombres y 106 en mujeres (razón de tasas 1.49, IC $95 \%$ 1,19-1,87). En las mujeres el mayor número de casos se notifica en las Actividades sanitarias y veterinarias. Y en los hombres, en la Construcción y en la Industria Manufacturera.

El número de casos notificados de hepatitis de transmisión sanguínea ha sido 90 en las mujeres y 30 en los hombres, lo que supone una tasa de 1,57 y 0,36 , respectivamente. El 88,89\% de los casos notificados en mujeres y el $66,67 \%$ de los casos en hombres se han producido en las Actividades sanitarias.

La tuberculosis, ha sido notificada en 37 mujeres y en 24 hombres, lo que supone una tasa de 0,65 y 0,29 respectivamente. Todos los casos notificados en mujeres y la mitad de los casos observados en hombres se producen en las Actividades sanitarias.

La sordera se ha notificado con mayor frecuencia en hombres, con una razón de tasas de 6,39 (IC 95\% 5,10-8,01). Los más afecta- dos son los trabajadores de la Fabricación de automóviles y los de la Metalurgia.

\section{DISCUSION}

En concordancia con lo descrito en otros trabajos ${ }^{13-17}$, en nuestros resultados hemos comprobado que las enfermedades que más se declaran corresponden a las enfermedades provocadas por agentes físicos, en particular por movimientos repetidos y posturas forzadas, en concreto las tenosinovitis de mano-muñeca, las epicondilitis y las bursitis de rodilla, procesos que podemos englobar en el gran grupo de enfermedades osteomusculares, que representan el $71 \%$ de todas las enfermedades profesionales declaradas en el año 2.004. Llama la atención que no se haya notificado ningún caso de patología osteomuscular distinta de las citadas. Según el estudio Valoración socioepidemiológica del paciente con dolor de espalda, realizado por la Sociedad Española del Dolor ${ }^{18}$, el dolor de espalda afecta a un $75 \%$ de las personas adultas a lo largo de su vida y en el $90 \%$ de los casos se debe a una alteración mecánica de las estructuras vertebrales. Esta patología es la causa más frecuente de la limitación de la actividad laboral en menores de 50 años, atribuyendo el $36 \%$ de las personas que padecen lumbalgia a su actividad laboral.

El segundo grupo de enfermedades profesionales más declaradas son las cutáneas, en su mayoría dermatitis, y el tercero las neurológicas, fundamentalmente el síndrome del túnel carpiano, encontrándose estos resultados dentro del rango descrito por otros autores $^{19}$. Llama la atención que sólo se han declarado 11 casos de cáncer (corresponden a 5 mesoteliomas, 2 leucemias, 1 cáncer de pulmón y 1 de laringe), y 13 enfermedades cardiovasculares.

Con relación al sexo, el número de enfermedades notificadas ha sido mayor en hombres que en mujeres, en una proporción de 2 a 1. Sin embargo, al comparar las inciden- 
cias calculadas por ocupación hemos visto que, a excepción de la bursitis de rodilla (cuya incidencia es muy baja tanto en hombres como mujeres), y la sordera, de claro predominio masculino, en muchas ocupaciones la tasa es más elevada en las mujeres: el túnel carpiano y las tenosinovitis son 7 veces y 5 veces más frecuentes respectivamente en las trabajadoras de las industrias manufactureras, por ejemplo, y la incidencia de hepatitis de transmisión sanguínea es 6 veces mayor en las profesionales sanitarias. Esto puede ser explicado por la segregación en la ocupación que hace que mujeres y hombres se concentren en tipos y niveles diferentes de actividad y de empleo, donde las mujeres se ven confinadas a una gama más estrecha de ocupaciones que los hombres (segregación horizontal), y a puestos de trabajo inferiores (segregación vertical ${ }^{20}, \mathrm{y}$ que, como decíamos en la introducción, exponen a mujeres y hombres a diferentes condiciones de trabajo y de vida ${ }^{6,7}$.

Por lo que se refiere a la actividad económica, hemos visto que, tanto en hombres como en mujeres, son las industrias extractivas y las manufactureras las actividades que presentan una mayor tasa de enfermedades profesionales, encontrando incidencias elevadas coherentes con los factores de riesgo propios de cada rama de actividad cuando las analizamos con más detalle. Sin embargo, llama poderosamente la atención la mínima declaración (o incluso nula) de determinadas enfermedades en determinados sectores y ocupaciones. Así sucede con el asma y las dermatitis de etiología alérgica, en sectores en los que sabemos hay alergenos. En este sentido, en Finlandia la tasa de incidencia de asma es siete veces mayor que en nuestro país ${ }^{21}$. La comparación de incidencias por sexo muestra otra vez diferencias que deberían ser objeto de estudios específicos. Hay determinadas enfermedades del aparato osteoarticular que predominan en las mujeres y en ciertos sectores feminizados, pero incluso en algunos sectores de predominio masculino (como la transformación del caucho y materias plásticas, la fabricación de material de transporte, la construcción de maquinaria y equipo mecánico) la incidencia es mayor en las mujeres, incluso cuando la razón de tasas global es mayor en hombres (epicondilitis).

Las diferencias territoriales siguen siendo marcadas, y son similares a las que se vienen describiendo en otros estudios ${ }^{22,23}$. Como se comenta en estos estudios, estas diferencias entre Comunidades Autónomas pueden deberse a lo que se ha llamado paradoja de la notificación ${ }^{24}$, que explicaría las altas incidencias de algunas Comunidades por su mejor sistema de notificación, con búsqueda activa de casos.

Otro aspecto relevante que hemos observado, aunque a priori no estaba contemplado como objetivo de este estudio, es que el sistema vigente de declaración y registro de enfermedades profesionales es poco sensible para recoger las enfermedades prevalentes hoy en día: multifactoriales, crónicas, con largos periodos de latencia, e inespecíficas. Para las enfermedades profesionales «clásicas», específicas y monocausales, el sistema funciona mejor, aunque su distribución por sectores de actividad económica y ocupación, hace pensar en motivos socioeconómicos como variable explicativa, además de en la asociación con factores de riesgo de origen laboral. Finalmente, ciertas enfermedades profesionales se dan con mayor incidencia en las mujeres de ciertos sectores y ocupaciones y, en otros, no se declaran en absoluto, lo que podría estar indicando que las distintas «formas « de trabajar de las mujeres, por un lado, y las distintas formas de declarar sus enfermedades, por otro, generan diferencias en la morbilidad de origen laboral de hombres y mujeres.

En cuanto a la utilización de la EPA como fuente de datos para obtener los denominadores de las tasas que se utilizan en este estudio, como es conocido, y dada la definición de enfermedad profesional, las estadísticas 
oficiales utilizan como denominador la población trabajadora afiliada a la Seguridad Social con esta contingencia profesional cubierta, pero esta población no se encuentra disponible desagregada por género, edad, actividad económica y ocupación, que son nuestras variables de interés en el análisis. Por este motivo, utilizamos como denominadores las poblaciones de la EPA sabiendo, además, que permiten un alto grado de validez de los índices así obtenidos. Efectivamente, en el análisis de las lesiones profesionales que periódicamente publica el Instituto Navarro de Salud Laboral ${ }^{13}$, se realiza la comparación de los índices de incidencia por sectores calculados con la población asalariada de la EPA y con la población afiliada a la Seguridad Social con contingencia cubierta. Esta comparación permite apreciar la validez de los índices obtenidos con población de la EPA, ya que, excepto para el sector agrícola, donde los datos extraídos de la EPA no contemplan el Régimen Especial Agrario Cuenta Propia, las curvas de evolución de los índices en el periodo 1992-2001 son prácticamente superponibles para el total y los otros tres sectores de actividad económica: los índices para Industria y Servicios son iguales durante todo el periodo, y hay una ligera diferencia desde 1995 en Construcción.

Otro aspecto metodológico a comentar es el relativo a la utilización de la población asalariada del mismo año en el que se declaran los casos incidentes de enfermedad (en nuestro caso el 2004) como denominador de las tasas que calculamos. Para las enfermedades con largos periodos de latencia (cáncer, sordera, respiratorias, etc.) no serviría. En estos casos, el denominador correcto a utilizar serían los trabajadores de hace 10 a 40 años, dependiendo de la enfermedad. Sobre todo, en sectores y ocupaciones que hayan sufrido reestructuraciones y/o reconversiones, lo que provocaría cambios en el número de trabajadores que pueden ser importantes. En este estudio, esta limitación solo resulta relevante para la sordera y qui- zás el asma, ya que, como hemos visto en los resultados, la mayor parte de las enfermedades reconocidas por el sistema son agudas. Efectivamente, la edad media de presentación de estas enfermedades así como el breve tiempo de exposición que hemos encontrado, nos están indicando que las enfermedades notificadas son las que tienen un periodo de latencia corto.

Por lo que se refiere a los resultados que hemos obtenido, el objetivo del sistema es la prestación económico-sanitaria de las enfermedades que son consideradas como profesionales por el sistema de Seguridad Social, no siendo ni su vigilancia epidemiológica ni su prevención objetivos del mismo ${ }^{1}$. El aprovechamiento de la información que se recoge es, por lo tanto, limitado para estos fines. A pesar de ello, consideramos que su análisis proporciona información de interés, tal y como hemos visto.

Hemos de destacar también que, si se analizan las enfermedades del Cuadro español una a una, se comprueba que muchos epígrafes de la lista no son objeto de declaración, y esta circunstancia puede deberse a que la sustancia o actividad no es muy frecuente o no existe en nuestro país, o que las medidas preventivas son muy eficaces, y la actividad no presenta riesgo, o que quizás simplemente no se declaran. Paralelamente, cada vez es más frecuente encontrarnos con enfermedades claramente profesionales, cuyos agentes o causas no están en la lista ${ }^{14}$. Se califican como accidentes de trabajo, si se demuestra su origen laboral, pero tras un gran esfuerzo del propio trabajador. Ante la próxima reforma del sistema vigente de declaración y registro de las enfermedades profesionales, estos aspectos deberían contemplarse e influir sobre el contenido de la nueva lista.

En conclusión, las enfermedades profesionales más declaradas en España en el año 2004 fueron las enfermedades osteomusculares (que acumulan las tres cuartas partes de los casos notificados), las neurológicas (fun- 
damentalmente túnel carpiano) y las cutáneas, en su mayoría dermatitis de contacto. Dentro del gran grupo de enfermedades osteomusculares, en las mujeres la más declarada es la tenosinovitis de mano-muñeca y, en los hombres, las epicondilitis. La diferencia más destacable encontrada entre hombres y mujeres es que, aunque la incidencia global es mayor en hombres que en mujeres, en la mayoría de actividades económicas y ocupaciones, la incidencia es mayor en la mujer. En las mujeres, las enfermedades profesionales se declaran en edades más tempranas que en los hombres. En el conjunto de procesos estudiados, la antigüedad en el puesto de trabajo fue menor en las mujeres. Son sobretodo las grandes empresas las que notifican enfermedades profesionales en las mujeres.

Sería deseable que en la próxima actualización del sistema vigente de declaración y registro de enfermedades profesionales se tuvieran en cuenta estos aspectos y se buscara su mejora. Imprescindible resulta que la publicación oficial periódica de las estadísticas de accidentes y enfermedades profesionales incluya el sexo como variable de análisis.

\section{BIBLIOGRAFÍA}

1. García Gómez M. Consideraciones sobre el sistema actual de declaración y registro de las enfermedades profesionales. Gac Sanit 1993; 34:46-50.

2. Ministerio de Sanidad y Consumo. Informe sobre la salud de los españoles:1998. Madrid: Ministerio de Sanidad y Consumo; 1999.

3. Burger EJ. Restructuring workers'compensation to prevent occupational disease. Ann NY Acad Sci 1989; 572: 282-283.

4. International Labour Office. World day for Safety and Health at Work 2005: a background paper. [2005]. Disponible en: www.ilo.org/public/spanish/index.htm

5. García AM, Gadea R. Estimación de la mortalidad y morbilidad por enfermedades laborales en España. Arch Prev Riesgos Labor 2004; 7(1):3-8.
6. Fundación Europea para la mejora de las Condiciones de Vida y de Trabajo. Encuesta Europea de Condiciones de Trabajo. [2005]. Disponible en: http://europa.eu.int/agencies/efound/index_es.htm

7. Almodóvar A, Nogareda C, Fraile A, De la Orden V, Zimmermann M, Villar MF, et al. V Encuesta Nacional de Condiciones de Trabajo. Madrid: Instituto Nacional de Seguridad e Higiene en el Trabajo; 2004.

8. Zimmermann Verdejo M, González Gómez MF, Martínez Vidal M. Las condiciones de trabajo según la perspectiva de género. Implicaciones y repercusiones sobre la morbilidad. La Mutua 2006; 14 (segunda época):91-103.

9. Real Decreto 917/1994, de 6 de mayo, por el que se aprueba la Clasificación Nacional de Ocupaciones 1994 (CNO-94). BOE núm 126 de 27/05/1994.

10. Real Decreto $1560 / 1992$, de 18 de diciembre, por el que se aprueba la Clasificación Nacional de Actividades Económicas (CNAE-93), BOE núm 306, de 22/12/1994

11. Organización Mundial de la Salud. Clasificación Estadística Internacional de Enfermedades y problemas relacionados con la salud. $10^{\text {a }}$ revision. Génova: Organización Mundial de la Salud; 2004.

12. Encuesta de Población Activa (EPA). Instituto Nacional de Estadística (INE). Madrid: Ministerio de Economía y Hacienda; 2004.

13. E Layana Echezuri, L Artieda Pellejero, M Lezáun Goñi, A Beloqui Basterra. Lesiones profesionales en Navarra 2001. Gobierno de Navarra. Instituto Navarro de Salud Laboral.

14. Comisión Nacional de Seguridad y Salud en el Trabajo. Enfermedades Profesionales. Madrid: Comisión Nacional de Seguridad y Salud en el Trabajo; 1999.

15. EUROSTAT. Occupational Diseases in Europe 2001. [2006]. Disponible en: http://epp.eurostat. cec.eu.int/cache/ITY_OFFPUB/KS-NK-04015/EN/KS-NK-04-015-EN.PDF

16. Agencia Europea para la Salud y la Seguridad en el Trabajo. Informe Anual de la Agencia 2004. Bilbao: Agencia Europea para la Salud y la Seguridad en el Trabajo; 2004.

17. Ordaz E, Maqueda J. Análisis de la incidencia y tendencias de las enfermedades profesionales en España (1998-2003). Med Segur Trab 2005; Vol LI (199):41-51. 
18. Sociedad Española del Dolor. Valoración socioepidemiológica del paciente con dolor de espalda. El Médico Interactivo 2005 Nov 20; Sec. Sociedades Científicas.

19. Cherry N. Recent advances. Occupational disease. BMJ 1999; 318:1397-9.

20. Comisión Europea. Dirección General de Empleo, Relaciones Laborales y Asuntos Sociales.100 palabras para la igualdad. Glosario de términos relativos a la igualdad entre hombres y mujeres. Bruselas: Comisión europea; 1998

21. Finnish Register of Occupational Diseases and the ASA Register. Occupational diseases in Finland in
1989. Reviews 16 Helsinki: Finnish Institute of Occupational Health Publication Office; 1998.

22. Instituto Navarro de Salud Laboral. Diagnóstico de Salud Laboral. Navarra. 1994-1996. Pamplona: Fondo de Publicaciones del Gobierno de Navarra; 1998.

23. Durán F, G. Benavides F. Informe de Salud Laboral. Los riesgos laborales y su prevención. España 2004. Barcelona: Atelier; 2004.

24. Rantanen J, Kauppinen T, Toikkanen J, Kurppa K, Lehtinen S, Leino T. Work and health country profiles. Country profiles and national surveillance indicators in occupational health and safety. People and work. Research Reports 44. Helsinki: Finnish Institute of Occupational Health Publication Office; 2001. 